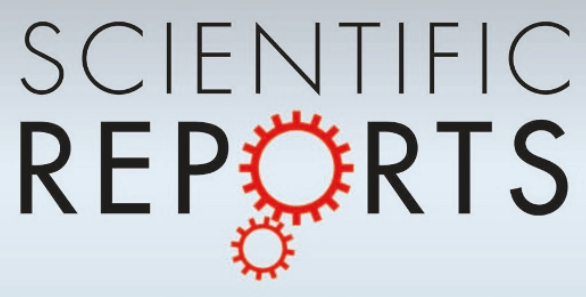

OPEN

SUBJECT AREAS:

MATERIALS SCIENCE

MATERIALS CHEMISTRY

MECHANICAL PROPERTIES

MECHANICAL AND STRUCTURAL

PROPERTIES AND DEVICES

Received

10 September 2012

Accepted

12 June 2013

Published

27 June 2013

Correspondence and requests for materials should be addressed to X.D.L. (lixiao@cec.sc.

edu)

\section{Enhancing graphene reinforcing potential in composites by hydrogen passivation induced dispersion}

\author{
Yingchao Yang, William Rigdon, Xinyu Huang \& Xiaodong Li
}

Department of Mechanical Engineering, University of South Carolina, 300 Main Street, Columbia, South Carolina 29208, USA.

To take full advantages of the structural uniqueness and exceptional properties of graphene as reinforcement in composites, harvesting well-dispersed graphene is essential. On the other hand, it is challenging to achieve simultaneously high stiffness, strength and toughness in engineered materials because of the trade-off relations between these properties. Here we demonstrate that the graphene reinforcing potential can be significantly enhanced through the excellent dispersion of graphene sheets in the matrix material and the strong graphene-matrix bonding by the coupled hydrogen passivation and ultrasonication technique. The fabricated graphene/epoxy composites exhibit simultaneously remarkable increase in elastic modulus, fracture strength, and fracture energy. We found that the inlet hydrogen atoms in the hydrogen passivation serve as a source of the second atoms to terminate the $\mathrm{C}$ dangling bonds and form more stable $\mathrm{C}-\mathrm{H}$ bonds, separating graphene flakes and promoting the binding with the matrix material.

- xceptional physical properties, high aspect ratio and low density of graphene have made it an ideal candidate for developing the next generation of polymer composites ${ }^{1-5}$. Graphene, as the strongest material ever measured, is known to have a Young's modulus of $1 \mathrm{TPa}$ and intrinsic strength of $130 \mathrm{GPa}$. Such values are 5 and 20 times greater than those for steel, respectively, at just $1 / 3$ the weight ${ }^{6-9}$. Thus, graphene sheets are expected to serve as mechanical reinforcements for lightweight composite systems. However, graphene has largely disappointed us in manufacturing lightweight, high strength polymer composites because of graphene sheet agglomeration in the matrix - one of the major roadblocks limiting its reinforcing effect and applications ${ }^{10,11}$. The mechanical properties of graphene reinforced polymer composites are considerably below their theoretically predicted potential $^{12}$. A common observation from previous studies is that the magnitude of improvement is, to a large extent, dependent upon the state of dispersion of graphene sheets in the matrix. Only when graphene sheets are homogeneously dispersed within the matrix, the enhancement could be more significant. Graphene sheets often aggregate into flakes of weakly interacting monolayered sheets due to its strong hydrophobicity and van der Waals attraction ${ }^{13}$. One flake can contain up to several hundred monolayered sheets ${ }^{14,15}$. The monolayered sheets within a flake can easily slide over each other and the shear modulus of graphene flakes is relatively low ${ }^{16}$. In addition, the aggregates of graphene sheets dramatically reduce the aspect ratio of the reinforcement.

It has been shown that harvesting well-dispersed graphene is challenging. Although chemical functionalization of graphene sheets may reduce their agglomeration tendency to some extent ${ }^{11,17-19}$, the major drawback of chemical surface modification is that impurity is often introduced into the graphene based composites and the presence of foreign stabilizers is undesirable and difficult to remove in further processes ${ }^{20}$. Many researchers have turned their attention to derivatives of graphite, especially graphite oxide, which is hydrophilic and has a larger interlayer distance than graphite ${ }^{21-24}$. Individual graphene oxide sheets can be produced in water more easily than monolayered graphene sheets, and such graphene oxide sheets may form stable dispersion after ultrasonication. Posterior deoxygenation is able to restore electrically insulating graphene oxide to conductive graphene, but many of the resultant sheets are crumpled and wrinkled ${ }^{25}$. New strategies to produce monolayered graphene sheets without destroying their unique structural integrity are in a pressing need. Surface passivation has been proven to be one of most effective methods to disperse multi-walled carbon nanotubes (MWCNTs) in both absolute ethanol and epoxy ${ }^{26}$. The inlet hydrogen atoms serve as a source of the second atoms to occupy the dangling C-bonds in MWCNTs, forming more stable $\mathrm{C}-\mathrm{H}$ bonds ${ }^{27}$. The dangling bonds provide graphene with high chemical activity and the possibility to uptake hydrogen ${ }^{28}$, which may be used to passivate the surface of graphene, thereby achieving excellent dispersion ${ }^{29}$. 
Here we limit our focus to graphene reinforced composites fabricated by the coupled hydrogen passivation (HP) and ultrasonication dispersion method. Atomic force microscopy (AFM), which has been proven to be the most effective method to visualize dispersed graphene sheets, was employed to evaluate the thickness of the graphene sheets after 2 hours of dispersion assisted by ultrasonication only and the coupled HP and ultrasonication, respectively. Threepoint bending tests of the graphene/epoxy composites fabricated by the coupled HP and ultrasonication technique revealed remarkable increases in elastic modulus, fracture strength, and fracture energy with an increase in graphene concentration. We demonstrate that the reinforcing limits of graphene composites can be further pushed up by the coupled HP and ultrasonication technique, which overcomes the roadblock and creates opportunities for taking advantages of the structural uniqueness and exceptional mechanical prowess of graphene in its applications.

\section{Results}

The raw graphene sheets used in this study were $12 \mathrm{~nm}$ in thickness. SEM images of original graphene at both low- and high-magnification can be seen in Figs. 1a and 1b, which illustrate that graphene sheets were bundled together. Figs. 1c and 1d show the SEM images of the graphene sheets dispersed by ultrasonication only and the coupled HP and ultrasonication, respectively. For dispersion with ultrasonication only (the inset in Fig. 1c), the majority of the graphene sheets tended to agglomerate and formed flakes in the ethanol solution. Fig. 1c is a low-magnification SEM image of such dispersed graphene. Several smaller graphene flakes assembled into a bigger graphene block. However, the graphene sheets dispersed by the coupled ultrasonication and HP (Fig. 1d) exhibit a completely different result. Such graphene sheets were well dispersed in the solution, and no graphene floccules were found in the ethanol solution. The inset in Fig. 1d shows a high-magnification SEM image of graphene dispersed by the combined HP and ultrasonication. The individual graphene layers observed with SEM appear transparent. The silicon wafer coated by coarse-grained Au was clearly observed through the covered graphene sheets. Evidently excellent dispersion of the graphene was achieved by the combined HP and ultrasonication. Graphene sheets with different concentrations were dispersed into absolute ethanol using the combined HP and ultrasonication. Details of the dispersion can be found in the TEM images (Figure S1, Supplementary materials).

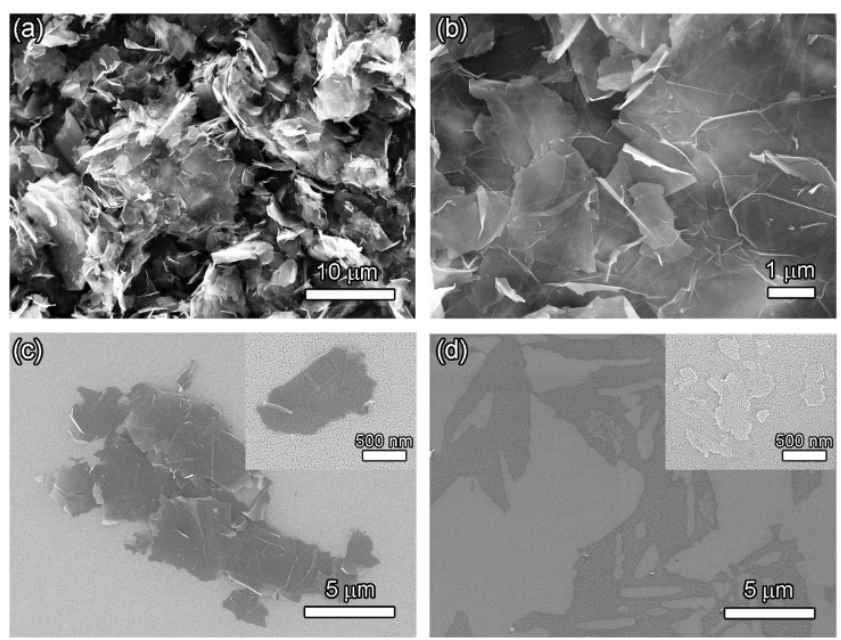

Figure 1 | SEM images of original and dispersed graphene sheets. (a) and (b) Low and high magnification images of original graphene sheets. (c) Low and high (insert) magnification images of graphene sheets dispersed by ultrasonication only. (d) Low and high (insert) magnification images of graphene sheets dispersed by the coupled HP and ultrasonication.
The graphene solutions dispersed by ultrasonication only and the combined HP and ultrasonication method were dropped onto silicon wafer and Mica for AFM observation, respectively. The AFM mounted with a silicon tip (tip radius: $8 \mathrm{~nm}$ ) was then employed to scan at random locations, and 50 AFM images were obtained. The average thickness of the dispersed graphene by ultrasonication only was $17.958 \pm 5.555 \mathrm{~nm}$ (Fig. 2a), which was even higher than the average thickness of the original graphene sheets before dispersion. Such difference implies that further agglomeration occurred during dispersion. The dispersed graphene was still in a block format, which is evident in the AFM cross-section analysis (Fig. 2c). However, the graphene dispersed by the coupled HP and ultrasonication gives rise to a rather different result. The solution containing dispersed graphene sheets was dropped onto the fresh surface of Mica, whose roughness is much lower than that of the silicon wafer. 50 random AFM images were obtained. The average thickness of the coupled HP and ultrasonication dispersed graphene was $1.275 \pm 0.607 \mathrm{~nm}$. A representative AFM image and corresponding cross-section profile of such dispersed graphene, with the thickness of $1.091 \mathrm{~nm}$, are shown in Figs. $2 \mathrm{~b}$ and $2 \mathrm{~d}$. The thickness of such dispersed graphene is much lower than that of the graphene dispersed by ultrasonication only. Fig. 2e shows the probability analysis of thickness distribution of the graphene sheets dispersed by ultrasonication only, versus the thickness distribution of the graphene sheets dispersed by the coupled HP and ultrasonication. The main peak of the graphene dispersed by the coupled HP and ultrasonication is located at $1 \mathrm{~nm}$, whereas a broad peak is found at $18 \mathrm{~nm}$ for the graphene dispersed by ultrasonication only. Clearly the coupled HP and ultrasonication method effectively disperses graphene and prevents its agglomeration, achieving well-dispersed graphene sheets in media.

Figure 3 shows the SEM images of the fresh cleavage surfaces of a pure epoxy resin (Fig. 3a) and the graphene/epoxy composites fabricated by ultrasonication only (Fig. $3 \mathrm{~b}$ ) and by the coupled HP and ultrasonication (Fig. 3c). The TEM images of the graphene sheets dispersed in epoxy can be found in Figure S2 in Supplementary materials. The fracture surface of the control sample is relatively smooth (Fig. 3a). Compared to the smooth fracture surface of the pure epoxy, the fracture surfaces of graphene/epoxy composites are rough and consist of many small facets, elucidating that the graphene inhibits fracture of the composites and thus results in a rougher fracture surface. However, the dispersion of graphene sheets by ultrasonication only was not good enough. Big graphene blocks can be seen in the graphene reinforced composites fabricated by ultrasonication only (Figs. $3 \mathrm{~b}$ and $3 \mathrm{~d}$ ). The big gap between the graphene sheets, as indicated by the arrow in Fig. $3 \mathrm{~d}$, implies that the graphene sheets slide over each other during the bending test. In this case, the graphene block actually worked as a defect in its composite, which would severely impair the mechanical properties of the composite. While the graphene sheets dispersed by the coupled HP and ultrasonication achieved excellent dispersion in epoxy matrix, no graphene blocks were found on the fracture surface. The graphene was found to be thickly coated with an adsorbed epoxy layer (Fig. 3e) and the graphene bridging was often seen on the fracture surface (Fig. 3f, and Fig. S3, Supplementary materials), jointly pointing toward strong epoxy-graphene interaction and enhanced mechanical properties.

\section{Discussion}

To unveil the HP induced dispersion mechanism, the chemical interaction between the graphene and the hydrogen atoms is propounded here. Graphene tends to form bundles due to the van der Waals interaction between individual monolayer graphene sheets and the formation of $\mathrm{C}-\mathrm{C}$ bonds by jointing $\mathrm{C}$ dangling bonds of neighboring graphene sheets ${ }^{28,29}$. Upon ultrasonication, graphene was agitated by the applied sound energy from ultrasonication. When the applied sound energy was high enough to overcome the van der Waals force 


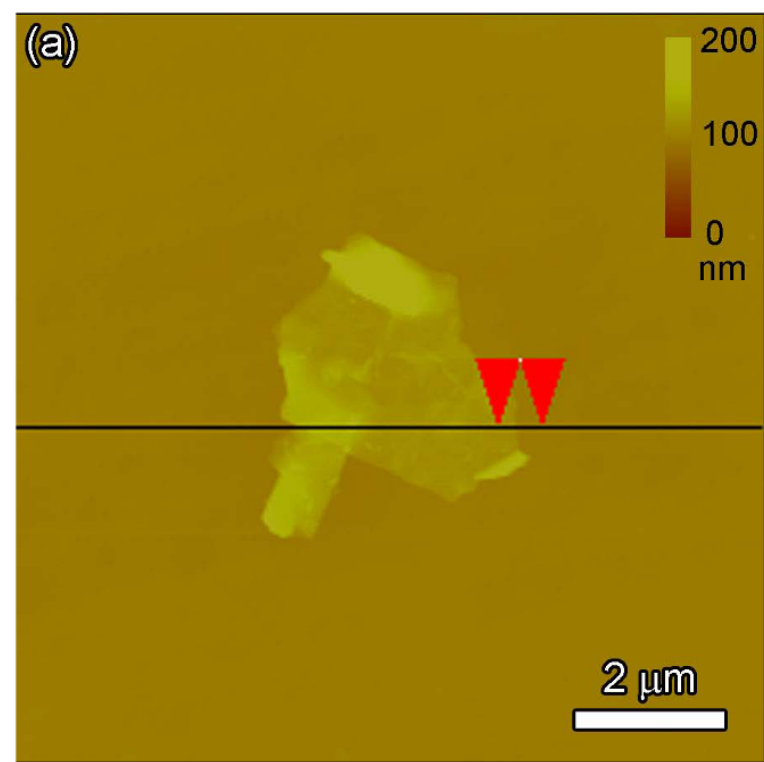

(c)

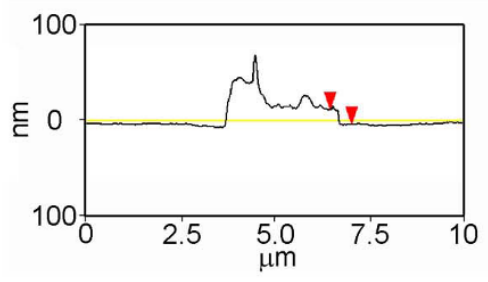

(d)

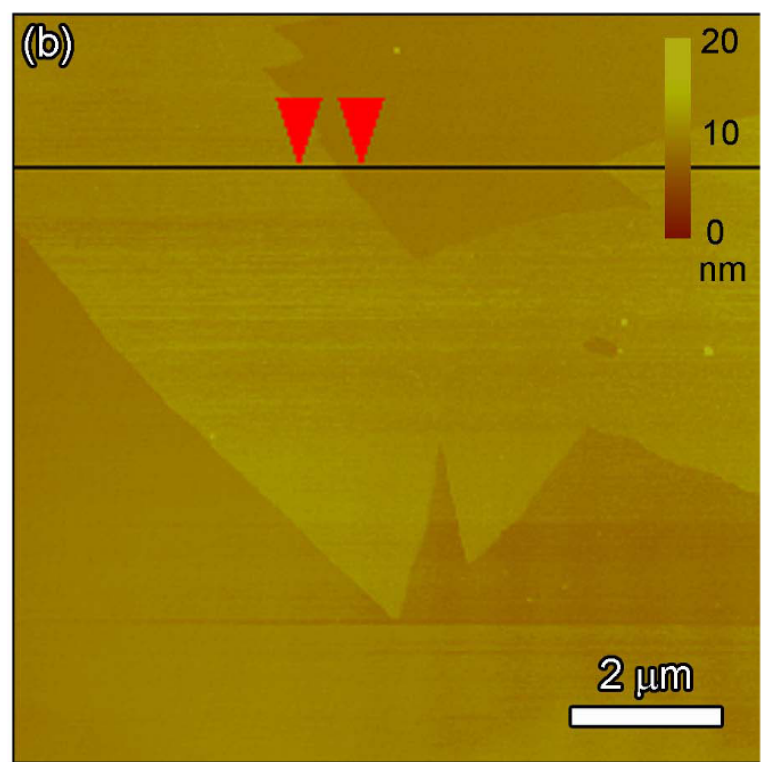

(e)
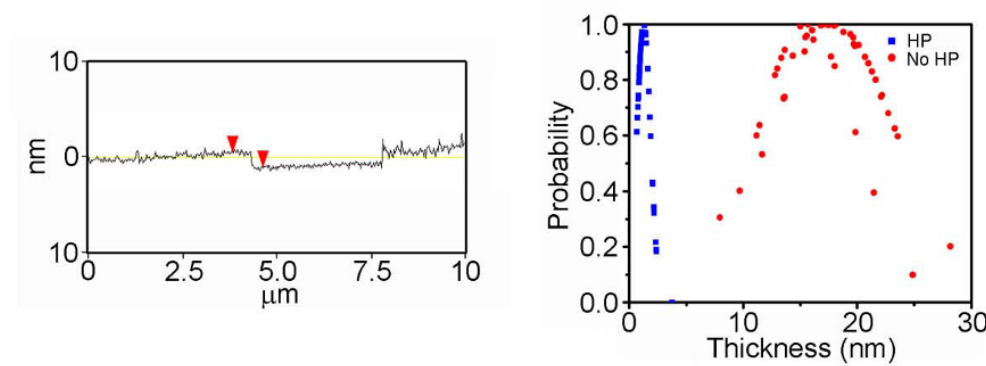

Figure $2 \mid$ AFM characterization on dispersed graphene sheets. (a) A representative AFM image of graphene sheets dispersed by ultrasonication only sitting on a silicon wafer. (b) A representative AFM image of graphene sheets dispersed by the coupled HP and ultrasonication sitting on Mica. (c) and (d) Cross sectional high profiles of graphene sheets in (a) and (b) indicated by line. (e) Probability of thickness distribution of graphene sheets dispersed by ultrasonication only, versus by the coupled HP and ultrasonication.

and break the $\mathrm{C}-\mathrm{C}$ bonds between the individual graphene sheets, the graphene bundles started to debond and be dispersed into the media. If there was no inlet hydrogen during the ultrasonication, the $\mathrm{C}$ dangling bonds resulted from the broken $\mathrm{C}-\mathrm{C}$ bonds might rebind with nearby $\mathrm{C}$ dangling bonds when the ultrasonication stopped, leading to rebundling of the graphene sheets. The inlet hydrogen then reacted with the $\mathrm{C}$ dangling bonds to form more stable $\mathrm{C}-\mathrm{H}$ bonds. This process passivated the graphene sheets and prevented them from bundling together through the formation of the $\mathrm{C}-\mathrm{H}$ bonds during and after ultrasonication so that the excellent dispersion of graphene was achieved. The inlet hydrogen atoms in the HP serve as a source of the second atoms to form more stable $\mathrm{C}-\mathrm{H}$ bonds. The following reaction occurs during the HP.

$$
\mathrm{C}-\mathrm{C}+\mathrm{H}_{2} \rightarrow 2 \mathrm{C}-\mathrm{H}
$$

Since the bond energy is $347 \mathrm{~kJ} / \mathrm{mol}$ for C-C, $436.2 \mathrm{~kJ} / \mathrm{mol}$ for $\mathrm{H}-\mathrm{H}$, and $416.3 \mathrm{~kJ} / \mathrm{mol}$ for $\mathrm{C}-\mathrm{H}$, it is clear that additional energy is needed to activate the reaction $(1)^{26}$. This additional energy is provided by the sound energy produced by the ultrasonication treatment. $\mathrm{C}-\mathrm{H}$ bonds can be identified by the chemical shift of the C1s level due to the hydrogen coordination change in $\mathrm{x}$-ray photoelectron spectroscopy (XPS). The plots (a) and (b) in Figure 4 show the C1s XPS for graphene sheets dispersed by ultrasonication only and by the couple HP and ultrasonication, respectively. The graphene was directly exposed to the hydrogen molecules during the HP. The hydrogen interaction with carbon dangling bonds leads to a dramatic change in the $\mathrm{C} 1$ s peak shape. The second $\mathrm{C} 1$ s peak is recognized as a shoulder at higher binding energy compared to the $\mathrm{C} 1 \mathrm{~s}$ peak of the graphene dispersed by ultrasonication only. This observed binding energy shift between the main peak and the second peak for the graphene dispersed by the couple HP and ultrasonication is attributed to a rehybridization from $s p^{2}$ to $s p^{3}$ due to the attachment of hydrogen to the $\mathrm{C}-\mathrm{C} \pi$ bonds, resulting in $\mathrm{C}-\mathrm{H}$ bond formation ${ }^{30,31}$. The combined HP and ultrasonication method to disperse graphene sheets has several unparalleled advantages over the conventional dispersion methods. The combined HP and ultrasonication method is powerful in dispersing graphene sheets. Unlike other surface modifications, in which graphene surface is functionalized by the reactions with atoms or molecules, such as organic groups, the HP adds no impurities that may affect the further application of the dispersed graphene. In fact, the hydrogen adsorbed in graphene can be released around $600^{\circ} \mathrm{C}^{31}$, therefore no impurities would remain in graphene.

The number of graphene layers was also measured by Raman spectroscopy (Horiba LabRam HR800 Raman Spectrometer). The solutions of graphene sheets with concentration of $1 \mathrm{mg} / \mathrm{ml} \mathrm{dis-}$ persed respectively by ultrasonication only and the combined HP and ultrasonication in absolute ethanol were dropped onto a silicon wafer and dried in ambient condition. Both $\mathrm{G}$ band and $2 \mathrm{D}$ band are given in Fig. 5. The $\mathrm{G}$ bands of the graphene sheets dispersed by ultrasonication only and the coupled HP and ultrasonication are at $1586.8 \mathrm{~cm}^{-1}$ and $1581.7 \mathrm{~cm}^{-1}$, respectively (Fig. 5a). Empirically, the $\mathrm{G}$ band position can be correlated to the number of atomic layers via the following relation ${ }^{32}$ : 

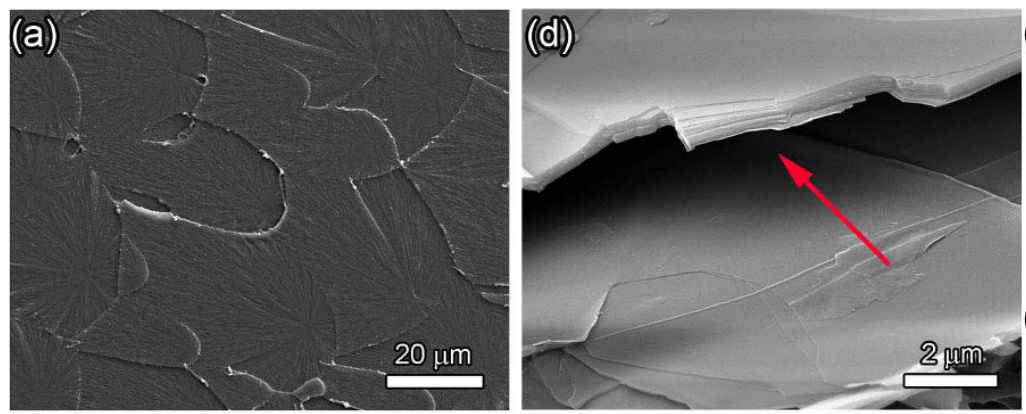

(g)
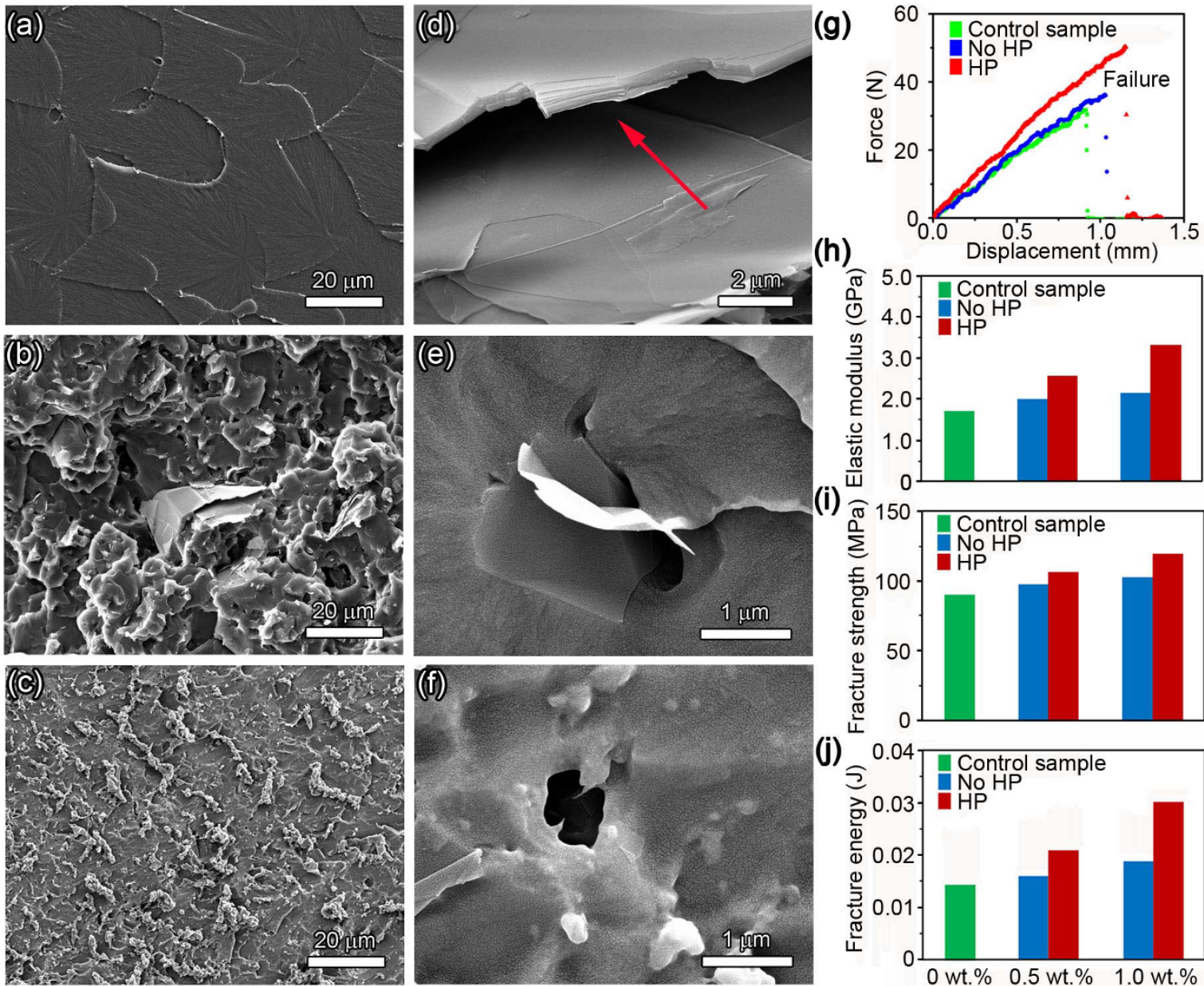

(h)

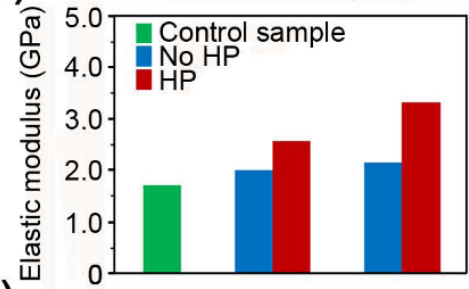

(i)

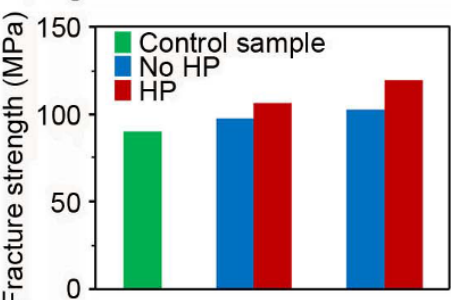

(j)

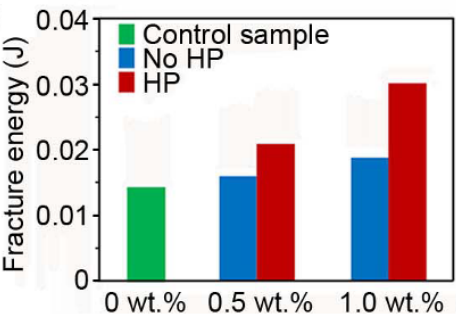

Figure $3 \mid$ Morphology and mechanical characterization of $1.0 \mathrm{wt} \%$ graphene/epoxy composites. (a-c) SEM images of fracture surfaces of pure epoxy resin, composites reinforced with graphene dispersed by ultrasonication only and by the coupled HP and ultrasonication, respectively. (d) High magnification image of graphene block in (b). (e) and (f) High magnification images of wrinkled and bridging graphene in (c). (g) Representative loaddisplacement curves. (h), (i), and (j) Variations of elastic modulus, fracture strength, and fracture energy with different graphene percentage for graphene/epoxy composites.

$$
\omega_{G}=1581.6+\frac{11}{1+n^{1.6}}
$$

The number of layers was found to be $2 \sim 5$ for the graphene sheets dispersed by the combined HP and ultrasonication, while the number of layers was calculated to be more than 60 for the graphene sheets dispersed by ultrasonication only. The second-order Raman $2 \mathrm{D}$ band reflecting the electronic band structure of graphene is shown in Fig. 5b. For multiple-layered graphene sheets, the 2D band is broad due to the change of electronic structure of graphene ${ }^{33}$. The number of layers of the graphene sheets dispersed respectively by ultrasonication only and coupled HP and ultrasonication was

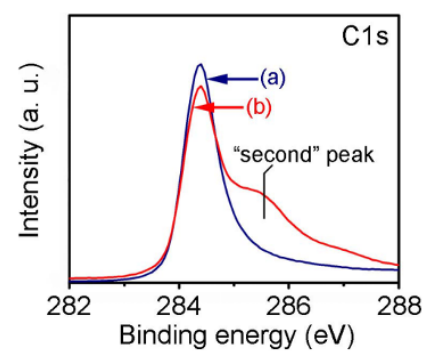

Figure $4 \mid$ C1s XPS of dispersed graphene. (a) Ultrasonication only and (b) coupled HP and ultrasonication.

determined to be more than 2 , as confirmed by both shape and position of the $2 \mathrm{D}$ band $^{34}$.

Three-point bending test was performed on the graphene/epoxy composites and epoxy control sample. The elastic modulus was calculated from the linear portion of the bending force-displacement (deflection) curve using the following equation ${ }^{35,36}$ :

$$
E=\frac{K L^{3}}{48 I}
$$

where $I, L$, and $K$ are the moment of inertia, the suspended length, and the slope of the initial linear portion of the bending forcedisplacement curve, respectively. The representative bending forcedisplacement curves are depicted in Fig. 3g. The bending force and displacement at failure for the graphene/epoxy composites fabricated by the coupled HP and ultrasonication were much higher than those of the control sample and the graphene/epoxy composites prepared by ultrasonication only. The elastic modulus values of the graphene/epoxy composites and the control sample determined by three-point bending are compared in Fig. 3h. As expected, the elastic modulus of the graphene/epoxy composites fabricated by either ultrasonication only or the coupled HP and ultrasonication increases with increasing graphene content. Specifically, the elastic modulus of the composites reinforced with $0.5 \mathrm{wt} . \%$ and 1 wt.\% graphene by ultrasonication only is, $1.81 \pm 0.38 \mathrm{GPa}$ and $1.95 \pm$ $0.42 \mathrm{GPa}$, respectively, demonstrating only $19.9 \%$ and $29.1 \%$ 

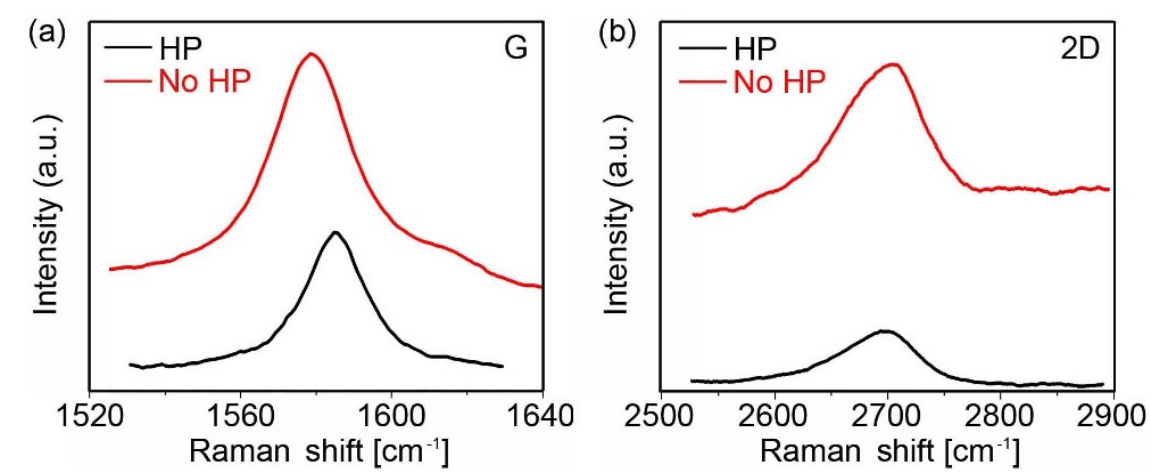

Figure 5 Raman spectra of the graphene sheets dispersed respectively by ultrasonication only and coupled HP and ultrasonication. (a) G band. (b) 2D band.

increase compared to the control sample with an elastic modulus of $1.51 \pm 0.15 \mathrm{GPa}$. Whereas the graphene/epoxy composites reinforced with the same amount of graphene fabricated by the coupled HP and ultrasonication exhibit a remarkable increase in elastic modulus. The elastic modulus of the $1.0 \mathrm{wt} . \%$ graphene reinforced epoxy composite was measured to be $3.07 \pm 0.21 \mathrm{GPa}$, demonstrating $103.3 \%$ and $57.4 \%$ increase in elastic modulus compared to the control sample and the composite with the same amount of graphene fabricated by ultrasonication only, respectively. The Halpin-Tsai model, which has been widely used for predicting the modulus of randomly distributed filler-reinforced nanocomposites ${ }^{37-40}$, was employed to calculate the theoretical elastic modulus of the composites by assuming that graphene sheets were perfectly dispersed in the matrix material and every monolayer graphene sheet was coherently bonded with the matrix. The composite modulus $E c$ is defined as

$$
\begin{gathered}
E_{c}=E_{m}\left[\frac{3}{8} \frac{1+\eta_{L} \xi V_{f}}{1-\eta_{L} V_{f}}+\frac{5}{8} \frac{1+2 \eta_{T} V_{f}}{1-\eta_{T} V_{f}}\right] \\
\eta_{L}=\frac{\frac{E_{f}}{E_{m}}-1}{\frac{E_{f}}{E_{m}}+\xi} \\
\eta_{T}=\frac{\frac{E_{f}}{E_{m}}-1}{\frac{E_{f}}{E_{m}}+2} \\
\xi=\frac{2 \alpha_{f}}{3}=\frac{2 l_{f}}{3 t_{f}}
\end{gathered}
$$

where $E_{c}$ is the predicted elastic modulus of composite, $E_{f}$ and $E_{m}$ are the elastic modulus of fiber and matrix, respectively. $V_{f}$ is the volume fraction of the fiber. $\alpha_{f}, l_{f}$, and $t_{f}$ refer to the aspect ratio, length, and thickness of the graphene sheet, respectively. The theoretical elastic modulus of the $1.0 \mathrm{wt} . \%$ graphene reinforced epoxy composite was calculated to be $3.56 \mathrm{GPa}$. In our study, the measured elastic modulus was up to $86.23 \%$ of the theoretical value. The increase in elastic modulus in the composites fabricated by the coupled HP and ultrasonication is higher than that in the composites with the same graphene contents in previous reports ${ }^{12,41-45}$.

Fracture strength, which is directly related to the flow strength of a material, depends on the effective load transfer between the matrix and the reinforcement phase in a composite. In this study, the fracture strength was calculated by the force at failure during bending using the following equation ${ }^{46}$ :

$$
\sigma_{f}=\frac{3 P L}{2 b d^{2}}
$$

where $P, L, b$, and $d$ are the maximum bending force, the suspended length, the width and height of the sample, respectively. The fracture strength of the control sample was measured to be $82.01 \pm 3.53 \mathrm{MPa}$, whereas the fracture strength of the graphene/epoxy composites reinforced with $1.0 \mathrm{wt}$ \% graphene fabricated by ultrasonication only and the combined HP and ultrasonication was increased up to 92.81 $\pm 1.47 \mathrm{MPa}$ and $121.34 \pm 5.48 \mathrm{MPa}$, respectively, demonstrating $13.2 \%$ and $47.9 \%$ increase compared to the control sample.

Fracture energy, which is the ability of a material to absorb energy and deform up to fracture, was obtained by integrating the loaddisplacement curve up to the fracture failure (Section 4, Supplementary materials). The fracture energy of the control sample, graphene/ epoxy composites reinforced with 1.0 wt.\% graphene fabricated by ultrasonication only and the coupled HP and ultrasonication, was measured to be $0.014 \pm 0.002 \mathrm{~J}, 0.019 \pm 0.002 \mathrm{~J}$, and $0.03 \pm 0.004 \mathrm{~J}$, respectively. The $1.0 \mathrm{wt} \%$ graphene reinforced composite fabricated by the coupled HP and ultrasonication exhibits $111.2 \%$ and $32.0 \%$ increases in fracture energy compared to the control sample and the composite with 1.0 wt.\% of graphene fabricated by ultrasonication only, respectively. Such remarkable, simultaneous increases in fracture strength and energy are ascribed to the excellent dispersion of graphene sheets in the epoxy matrix and the strong interfacial bonding between the epoxy and the well-dispersed hydrogen-passivated graphene (Fig. 3e). If the interface debonds easily, the load transfer will fail ${ }^{47}$. For instance, the graphene block in the composite fabricated by ultrasonication only (Fig. 4d) debonded and slid over each other, leading to fracture failure at much lower loads. Whereas the composites fabricated by the coupled HP and ultrasonication method demonstrate superior dispersion and ultra strong interfacial bonding, as evidently seen in the wrinkled texture fracture surface and individual graphene sheets wrapped with epoxy on the fracture surface (Figs. 3e and 3f), which is believed to be responsible for providing an effective load transfer from the epoxy matrix to individual graphene sheets to achieve the exceptional combined properties of high strength, stiffness and fracture energy.

In summary, a simple, low-cost technique that combines HP with ultrasonication has been developed to separate graphene flakes with an excellent dispersion in both absolute ethanol solution and epoxy resin, overcoming the major roadblock in the application of graphene. The yield is based on the quantity of hydrogen introduced into the solution and the power of ultrasonication. The fabricated graphene/epoxy composites exhibit simultaneous increases in elastic modulus, fracture strength, and fracture energy. The elastic modulus, fracture strength, and fracture energy values of the $1.0 \mathrm{wt} \%$ graphene/epoxy composites fabricated by the coupled HP and ultrasonication are $3.07 \pm 0.84 \mathrm{GPa}, 121.34 \pm 5.48 \mathrm{MPa}$, and $0.030 \pm$ $0.004 \mathrm{~J}$, respectively, demonstrating respective $103.3 \%, 47.9 \%$, and $111.2 \%$ increase compared to those of the matrix material. The coupled HP and ultrasonication method should find more 
applications where the structural uniqueness and exceptional properties of graphene need to be fully utilized.

\section{Methods}

Dispersion of graphene sheets in absolute ethanol. The average flake thickness of the graphene sheets (Graphene Supermarket, NY, USA) used in this study was $12 \mathrm{~nm}$. The specific surface area was about $80 \mathrm{~m}^{2} / \mathrm{g}$. To disperse the graphene sheets in absolute ethanol, graphene sheets $(\sim 1 \mathrm{mg})$ were first immersed into ethanol $(1 \mathrm{ml}$, Sigma-Aldrich) in a glass bottle. Then the glass bottle was put into the basin of an ultrasonicator (Branson 5510, Branson Ultrasonic Corp., CT, USA). Ultrasoncation was carried out in two ways, namely with the inlet hydrogen (HP) and in ambient environment. The hydrogen gas was produced by the reaction of zinc powder (SigmaAldrich) and hydrochloric acid (0.1 mol/L, Fisher Corp.). This gas passed through a long steel tube to eliminate any hydrochloric acid vapor.

Measurement of the thickness of graphene sheets. A silicon wafer was cleaned by acetone and ethanol successively in an ultrasonicator. Each process was performed for $5 \mathrm{~min}$. A drop of the ethanol solution containing dispersed graphene sheets without HP was then dropped onto the silicon wafer. Another drop of the ethanol solution containing dispersed graphene sheets with HP was dropped onto the fresh surface of Mica (Jupiter Commercial Company, Kolkata, India). The thickness of dispersed graphene sheets by both ultrasonication only and coupled HP and ultrasonication was measured by a Veeco Dimension 3100 AFM (Veeco Metrology Group, NY, USA) with a tapping mode silicon tip. 50 images obtained at random locations were used to analyze the thickness distribution for both dispersion methods. Scanning electron microscopy (SEM; Zeiss Ultra plus Field Emission Gun (FEG)-SEM) was used to examine the dispersed graphene sheets on silicon and Mica substrates. To determine the C-C and C-H bonds, X-ray photoelectron spectroscopy (XPS; Kratos Axis Ultra DLD) was employed to characterize the graphene sheets dispersed with and without HP. The number of graphene layers was measured by Raman spectroscopy (Horiba LabRam HR800 Spectrometer).

Fabrication and mechanical characterization of graphene/epoxy composites. To produce graphene/epoxy composites, graphene sheets were first dispersed in hardener, EPO-TEK 302-3M Part B, (Epoxy Technology, MA, USA) with $2 \mathrm{~h}$ ultrasonication under HP. Then the epoxy resin, EPO-TEK 302-3M Part A, (Epoxy Technology, MA, USA) was added to the hardener and the mixture was stirred for 30 minutes and cured for 24 hours under ambient condition. The weight proportion of epoxy resin to hardener was set to 100 to 45 as suggested by the manufacturer. Four kinds of epoxy composites reinforced by $0.5 \mathrm{wt} . \%$ and $1.0 \mathrm{wt} . \%$ graphene sheets with and without HP treatment were fabricated in this study. A control sample without any graphene was also prepared for comparison. Fresh cleavage surfaces of both graphene/epoxy composites and the control sample were observed using SEM to examine the morphology and dispersion of the graphene sheets. To measure the elastic modulus and fracture strength of the graphene/epoxy composites, three-point bending tests were performed on all four kinds of the composites reinforced with 0.5 wt.\% and 1.0 wt.\% graphene sheets with and without HP treatment using a microtribometer (CETR Inc., CA, USA). Details of three-point bending test and various working applications of microtribometer can be found in Section 5 , Supplementary materials. The microtribometer has the load and displacement sensors that allow us to perform tensile and bending on the graphene/epoxy composite samples. The control sample was also tested for comparison. For each composite, five beam samples with the dimension of $20 \times 2.5 \times 2.0 \mathrm{~mm}$ (length $\times$ width $\times$ height) were tested. The elastic modulus of the tested composites was calculated from the initial linear portion of the three-point bending loading-displacementcurve. The loading force at failure in bending test was used to calculate the fracture strength of the beam.

1. Xia, F. N., Mueller, T., Lin, Y. M., Valdes-Garcia, A. \& Avouris, P. Ultrafast graphene photodetector. Nat. Nanotechnol. 4, 839-843 (2009).

2. Geim, A. K. \& Novoselov, K. S. The rise of graphene. Nat. Mater. 6, 183-191 (2007).

3. Meric, I. et al. Current saturation in zero-bandgap, top-gated graphene field-effect transistors. Nat. Nanotechnol. 3, 654-659 (2008).

4. Balandin, A. A. et al. Superior thermal conductivity of single-layer graphene. Nano Lett. 8, 902-907 (2008).

5. Huang, X., Qi, X. Y., Boey, F. \& Zhang, H. Graphene-based composites. Chem.Soc. Rev. 41, 666-686 (2012).

6. Lee, C. G., Wei, X. D., Kysar, J. W. \& Hone, J. Measurement of the elastic properties and intrinsic strength of monolayer graphene. Science 321, 385-388 (2008).

7. Stankovich, S. et al. Graphene-based composite materials. Nature 442, 282-286 (2006).

8. Chawla, N. \& Deng, X. Microstructure and mechanical behavior of porous sintered steels. Mater. Sci. Eng. A 390, 98-112 (2005).

9. Li, X. D. et al. Nanomechanical characterization of single-walled carbon nanotube reinforced epoxy composites. Nanotechnology 15, 1416-1423 (2004).

10. Li, D., Müller, M. B., Gilje, S., Kaner, R. B. \& Wallace, G. G. Processable aqueous dispersions of graphene nanosheets. Nat. Nanotechnol. 3, 101-105 (2008).

11. Zhou, X. S. et al. Dispersion of graphene sheets in ionic liquid $[\mathrm{bmim}]\left[\mathrm{PF}_{6}\right]$ stabilized by an ionic liquid polymer. Chem. Commun. 46, 386-388 (2010).
12. Fang, M., Wang, K. G., Lu, H. B., Yang, Y. L. \& Nutt, S. Covalent polymer functionalization of graphene nanosheets and mechanical properties of composites. J. Mater. Chem. 19, 7098-7105 (2009).

13. Tjong, S. C. Polymer nanocomposite bipolar plates reinforced with carbon nanotubes and graphite nanosheets. Energy Environ. Sci. 4, 605-626 (2011).

14. Shahil, K. M. F. \& Balandin, A. A. Graphene-multilayer graphene nanocomposites as highly efficient thermal interface materials. Nano Lett. 12, 861-867 (2012).

15. Ramanathan, T. et al. Functionalized graphene sheets for polymer nanocomposites. Nat. Nanotechnol. 3, 327-331 (2008).

16. Tsuji, T. \& Yamanaka, K. Observation by ultrasonic atomic force microscopy of reversible displacement of subsurface dislocation in highly oriented pyrolytic graphite. Nanotechnology, 12, 301-307 (2001).

17. Patil, A. J., Vickery, J. L., Scott, T. B. \& Mann, S. Aqueous stabilization and selfassembly of graphene sheets into layered bio-nanocomposites using DNA. Adv. Mater. 21, 3159-3164 (2009)

18. Zhang, X. Y. et al. Dispersion of graphene in ethanol using a simple solvent exchange method. Chem. Commun. 46, 7539-7541 (2010).

19. Boukhvalov, D. W. \& Katsnelson, M. I. Chemical functionalization of graphene with defects. Nano Lett. 8, 4373-4379 (2008).

20. Choi, W. \& Lee, J. W. Graphene: Synthesis And Applications. CRC Press. Boca Raton, FL. P37 (2012).

21. Si, Y. C. \& Samulski, E. T. Synthesis of water soluble graphene. Nano Lett. 8 , 1679-1682 (2008).

22. Medhekar, N. V., Ramasubramaniam, A., Ruoff, R. S. \& Shenoy, V. B. Hydrogen bond networks in graphene oxide composite paper: structure and mechanical properties. ACS Nano 4, 2300-2306 (2010).

23. Lu, J. et al. One-pot synthesis of fluorescent carbon nanoribbons, nanoparticles, and graphene by the exfoliation of graphene in ionic liquids. ACS Nano 3 , 2367-2375 (2009).

24. An, X. H. et al. Stable aqueous dispersions of noncovalently functionalized graphene from graphite and their multifunctional high-performance applications. Nano Lett. 10, 4295-4301 (2010).

25. Kim, H., Abdala, A. A. \& Macosko, C. W. Graphene/polymer nanocomposites. Macromolecules, 43, 6515-6530 (2010).

26. Yang, Y. C., Xu, Z. H., Pan, Z. W. \& Li, X. D. Hydrogen passivation induced dispersion of multi-walled carbon nanotubes. Adv. Mater. 24, 881-885 (2012).

27. Nieto-Márquez, A., Romero, R., Romero, A. \& Valverde, J. L. Carbon nanospheres: synthesis, physicochemical properties and applications. J. Mater. Chem. 21, 1664-1672 (2011).

28. Akhukov, M. A., Fasolino, A., Gornostyrev, Y. N. \& Katsnelson, M. I. Dangling bonds and magnetism of grain boundaries in graphene. Phys. Rev. B 85, 115407 (2012).

29. Song, L. L., Zheng, X. H., Wang, R. L. \& Zeng, Z. Dangling bond states, edge magnetism, and edge reconstruction in pristine and $\mathrm{B} / \mathrm{N}$-terminated zigzag graphene nanoribbons. J. Phys. Chem. C 114, 12145-12150 (2010).

30. Okpalugo, T. I. T., Papakonstantinou, P., Murphy, H., McLaughlin, J. \& Brown, N. M. D. High resolution XPS characterization of chemical functionalized MWCNTs and SWCNTs. Carbon 43, 153-161 (2005).

31. Nikitin, A. et al. Hydrogenation of single-walled carbon nanotubes. Phys. Rev. Lett. 95, 225507 (2005).

32. Wang, H., Wang, Y. F., Cao, X. W., Feng, M. \& Lan, G. X. Vibrational properties of graphene and graphene layers. J. Raman Spectrosc. 40, 1791-1796 (2009).

33. Ferrari, A. C. et al. Raman spectrum of graphene and graphene layers. Phys. Rev. Lett. 97, 187401 (2006).

34. Ni, Z. H. et al. Graphene thickness determination using reflection and contrast spectroscopy. Nano Lett. 7, 2758-2763 (2007).

35. Oliver, W. C. \& Pharr, G. M. An improved technique for determining hardness and elastic modulus using load and displacement sensing indentation experiments. J. Mater. Res. 7, 1564-1583 (1992).

36. Yang, Y. C., Wang, G. F. \& Li, X. D. Water molecule-induced stiffening in $\mathrm{ZnO}$ nanobelts. Nano Lett. 11, 2845-2848 (2011).

37. Liang, J. J. et al. Molecular-level dispersion of graphene into poly(vinyl alcohol) and effective reinforcement of their nanocomposites. Adv. Funct. Mater. 19, 2297-2302 (2009).

38. King, J. A., Klimek, D. R., Miskioglu, I. \& Odegard, G. M. Mechanical properties of graphene nanoplatelet/epoxy composites. J. Appl. Polym. Sci. 128, 4217-4223 (2013).

39. Mahmoud, W. E. Morphology and physical properties of poly(ethylene oxide) loaded graphene nanocomposites prepared by two different techniques. Eur. Polym. J. 47, 1534-1540 (2011).

40. Halpin, J. C. \& Kardos, J. L. Halpin-Tsai equations - review. Polym. Eng. Sci. 16, 344-352 (1976)

41. Das, B., Prasad, K. E., Ramamurty, U. \& Rao, C. N. R. Nano-indentation studies on polymer matrix composites reinforced by few-layer graphene. Nanotechnology $\mathbf{2 0}$, 125705 (2009).

42. Yasmin, A., Luo, J. J. \& Daniel, I. M. Processing of expanded graphite reinforced polymer nanocomposites. Compos. Sci. Technol. 66, 1182-1189 (2006).

43. Kuilla, T. et al. Recent advances in graphene based polymer composites. Prog Polym. Sci. 35, 1350-1375 (2010).

44. Vadukumpully, S., Paul, J., Mahanta, N. \& Valiyaveettil, S. Flexible conductive graphene/poly(vinyl chloride) composite thin films with high mechanical strength and thermal stability. Carbon 49, 198-205 (2011). 
45. Wang, X. L., Bai, H., Yao, Z. Y., Liu, A. R. \& Shi, G. Q. Electrically conductive and mechanically strong biomimetic chitosan/reduced graphene oxide composite films. J. Mater. Chem. 20, 9032-9036 (2010).

46. Sundararajan, S., Bhushan, B., Namazu, T. \& Isono, Y. Mechanical property measurements of nanoscale structures using an atomic force microscope. Ultramicroscopy, 91, 111-118 (2002).

47. Rafiee, M. A. et al. Fracture and fatigue in graphene nanocomposites. Small $\mathbf{6}$, 179-183 (2010).

\section{Acknowledgements}

Financial support for this study was provided by the U.S. National Science Foundation (CMMI-1129979 and CMMI-0968843). The authors thank the staff members at the

University of South Carolina EM Center for SEM technical support and Yating Mao for providing Mica substrate.

\section{Author contributions}

Y.Y. and X. L. designed the project. Y.Y. performed the experiments. Y.Y., W.R. and X.H. carried out the Raman spectroscopy experiments and analyzed the Raman results. Y.Y. and X.L. wrote this paper.

\section{Additional information}

Supplementary information accompanies this paper at http://www.nature.com/ scientificreports

Competing financial interests: The authors declare no competing financial interests. How to cite this article: Yang, Y.C., Rigdon, W., Huang, X.Y. \& Li, X.D. Enhancing graphene reinforcing potential in composites by hydrogen passivation induced dispersion. Sci. Rep. 3, 2086; DOI:10.1038/srep02086 (2013).

(c) (1) () $\odot$ This work is licensed under a Creative Commons AttributionBy No NonCommercial-NoDerivs 3.0 Unported license. To view a copy of this license, visit http://creativecommons.org/licenses/by-nc-nd/3.0 